\title{
Conventional superconductivity and charge-density-wave ordering in $\mathrm{Ba}_{1-x} \mathrm{Na}_{x} \mathrm{Ti}_{2} \mathrm{Sb}_{2} \mathrm{O}$
}

\author{
Fabian von Rohr, ${ }^{1,2, *}$ Andreas Schilling, ${ }^{1}$ Reinhard Nesper, ${ }^{2}$ Chris Baines, ${ }^{3}$ and Markus Bendele ${ }^{4}$ \\ ${ }^{1}$ Physik-Institut der Universität Zürich, Winterthurerstrasse 190, CH-8057 Zürich, Switzerland \\ ${ }^{2}$ Laboratory of Inorganic Chemistry, ETH Zürich, Wolfgang-Pauli-Straße 10, CH-8093 Zürich, Switzerland \\ ${ }^{3}$ Laboratory for Muon Spin Spectroscopy, Paul Scherrer Institut, CH-5232 Villigen PSI, Switzerland \\ ${ }^{4}$ Dipartimento di Fisica, Università di Roma "La Sapienza", Piazzale Aldo Moro 2, 00185 Roma, Italy \\ (Received 22 August 2013; revised manuscript received 13 September 2013; published 1 October 2013)
}

\begin{abstract}
We have investigated the low-temperature physical properties of $\mathrm{BaTi}_{2} \mathrm{Sb}_{2} \mathrm{O}$ and $\mathrm{Ba}_{1-x} \mathrm{Na}_{x} \mathrm{Ti}_{2} \mathrm{Sb}_{2} \mathrm{O}(x=0.05$, $0.1,0.15,0.2,0.25,0.3)$ by means of muon spin rotation $(\mu \mathrm{SR})$ and SQUID magnetometry. Our measurements reveal the absence of magnetic ordering below $T_{\mathrm{DW}}=58 \mathrm{~K}$ in the parent compound. Therefore the phase transition at this temperature observed by magnetometry is most likely due to the formation of a charge-denisty wave (CDW). Upon substitution of barium by sodium in $\mathrm{Ba}_{1-x} \mathrm{Na}_{x} \mathrm{Ti}_{2} \mathrm{Sb}_{2} \mathrm{O}$ we find for $x=0.25$ superconductivity with a maximum $T_{c}=5.1 \mathrm{~K}$ in the magnetization and a bulk $T_{c \text {,bulk }}=4.5 \mathrm{~K}$ in the $\mu \mathrm{SR}$ measurements. The temperature dependency of the London penetration depth $\lambda^{-2}(T)$ of the optimally doped compound can be well explained within a conventional weak-coupling scenario in the clean limit.
\end{abstract}

\section{INTRODUCTION}

Nesting at the Fermi surface is known to be a key feature for the occurrence of either charge- (CDW) or spin-density-wave (SDW) ordering, and it is considered to be of importance for the emergence of superconductivity in some materials [e.g., $\mathrm{BaFe}_{2-x} \mathrm{Co}_{x} \mathrm{As}_{2}$ (Ref. 1)]. The competition or coexistence of superconductivity and SDW ordering is one of the most extensively discussed topics for iron-based superconductors and for the stripe phases of cuprates. ${ }^{2,3}$ The competition or coexistence between superconductivity and CDW ordering at low temperatures is less often encountered [see, e.g., in $\mathrm{Cu}_{x} \mathrm{TiSe}_{2}$ (Ref. 4), in 2H-NbSe 2 (Ref. 5), and in $\mathrm{Ba}_{1-x} \mathrm{~K}_{x} \mathrm{BiO}_{3}$ (Refs. 6 and 7)], though the development of CDW order at zero field in the normal state of superconducting $\mathrm{YBa}_{2} \mathrm{Cu}_{3} \mathrm{O}_{6.67}$ has been prominently discussed. ${ }^{8}$

The large family of stacked, layered titanium oxide pnictide compounds were long considered as potential host structures for superconductivity. ${ }^{9}$ Most of these materials were identified to undergo magnetic or density wave (DW) ordering transitions at low temperatures, in the absence of superconductivity. For example, $\mathrm{Na}_{2} \mathrm{Ti}_{2} \mathrm{As}_{2} \mathrm{O}$ and $\mathrm{Na}_{2} \mathrm{Ti}_{2} \mathrm{Sb}_{2} \mathrm{O}$, which crystallize in a modified anti- $\mathrm{K}_{2} \mathrm{NiF}_{4}$-type structure, were found to undergo transitions to SDW-ordered states at $T_{\mathrm{SDW}}$ of $320 \mathrm{~K}$ and $115 \mathrm{~K}$, respectively. ${ }^{10}$

$\mathrm{BaTi}_{2} \mathrm{Sb}_{2} \mathrm{O}$ belongs to this family of compounds. Its structure consists of titanium, octahedrally surrounded by oxygen, leading to square planar $\mathrm{Ti}_{2} \mathrm{O}$ sheets. ${ }^{11}$ This compound was found to undergo a phase transition to a SDW or $\mathrm{CDW}$ around $T_{\mathrm{DW}}=55 \mathrm{~K}$ and it was proposed that below $T_{c}=1 \mathrm{~K}$ it is a superconductor. ${ }^{12}$ Recently, it was shown that upon substitution of barium by sodium in $\mathrm{Ba}_{1-x} \mathrm{Na}_{x} \mathrm{Ti}_{2} \mathrm{Sb}_{2} \mathrm{O}, T_{\mathrm{DW}}$ is lowered and eventually suppressed, while superconductivity reaches a maximum $T_{c}$ of approximately $5 \mathrm{~K}^{13}$

In this Rapid Communication we will show by a series of SQUID magnetometry and muon-spin rotation ( $\mu \mathrm{SR})$ experiments that $\mathrm{Ba}_{1-x} \mathrm{Na}_{x} \mathrm{Ti}_{2} \mathrm{Sb}_{2} \mathrm{O}$ is another example for the coexistence and competition of the periodic modulations of CDW and superconductivity. Our results suggest that the
CDW ordering competes with a conventional superconducting state in these materials.

\section{EXPERIMENTAL}

Standard solid-state reactions were employed to synthesize polycrystalline samples of $\mathrm{Ba}_{1-x} \mathrm{Na}_{x} \mathrm{Ti}_{2} \mathrm{Sb}_{2} \mathrm{O}$ with $x=0$, $0.05,0.10,0.15,0.20,0.25$, and $0.30 . \mathrm{BaO}(99.99 \%), \mathrm{BaO}_{2}$ (95\%), $\mathrm{Na}_{2} \mathrm{O}_{2}$ (95\%), Ti (99.99\%), and $\mathrm{Sb}$ (99.999\%) were mixed and pressed into pellets in an argon-filled glove box. The pellets were sealed in argon-filled niobium ampules and then sintered at $1000{ }^{\circ} \mathrm{C}$ for $24 \mathrm{~h}$. Then the samples were reground under inert atmosphere, repelletized, and sintered again for $36 \mathrm{~h}$ at $1000{ }^{\circ} \mathrm{C}$. The purity, symmetry, and cell parameters were checked by $\mathrm{x}$-ray powder diffraction using a Stoe STADIP diffractometer $\left(\mathrm{Cu}-\mathrm{K}_{\alpha 1}\right.$ radiation, $\lambda=1.54051 \AA$, Ge monochromator).

The magnetic properties were studied using a Quantum Design Magnetic Properties Measurement System (MPMS $\mathrm{XL}$ ) equipped with a reciprocating sample option (RSO). Transverse-field (TF) and zero-field (ZF) $\mu$ SR experiments were carried out at the LTF instrument at the $\pi \mathrm{M} 3$ beamline, and at the Dolly instrument at the $\pi \mathrm{E} 1$ beamline at the Paul Scherrer Institute (PSI), Switzerland. The superconducting pellets were cooled from above $T_{c}$ in a field of $\mu_{0} H=35 \mathrm{mT}$ for the TF experiments. The occurrence of magnetism was investigated in these samples with the ZF experiments. The $\mu$ SR time spectra have been analyzed using the free software package MUSRFIT. ${ }^{14}$

\section{RESULTS AND DISCUSSION}

In Fig. 1(a) we show magnetization $M(T)$ data of the parent compound $\mathrm{BaTi}_{2} \mathrm{Sb}_{2} \mathrm{O}$, in a field of $\mu_{0} H=1.0 \mathrm{~T}$, showing a distinct kink at $T_{\mathrm{DW}}=58 \mathrm{~K}$. This discontinuity was earlier attributed to either a SDW or a CDW ordering transition. ${ }^{12}$

The ZF and weak TF muon time signals for $\mathrm{BaTi}_{2} \mathrm{Sb}_{2} \mathrm{O}$ were measured above and below $T_{\mathrm{DW}}$ at $T=1.5 \mathrm{~K}$ and $T=100 \mathrm{~K}$, as shown in Figs. 1(b) and 1(c). The measurements show neither indications of static nor fluctuating magnetism 

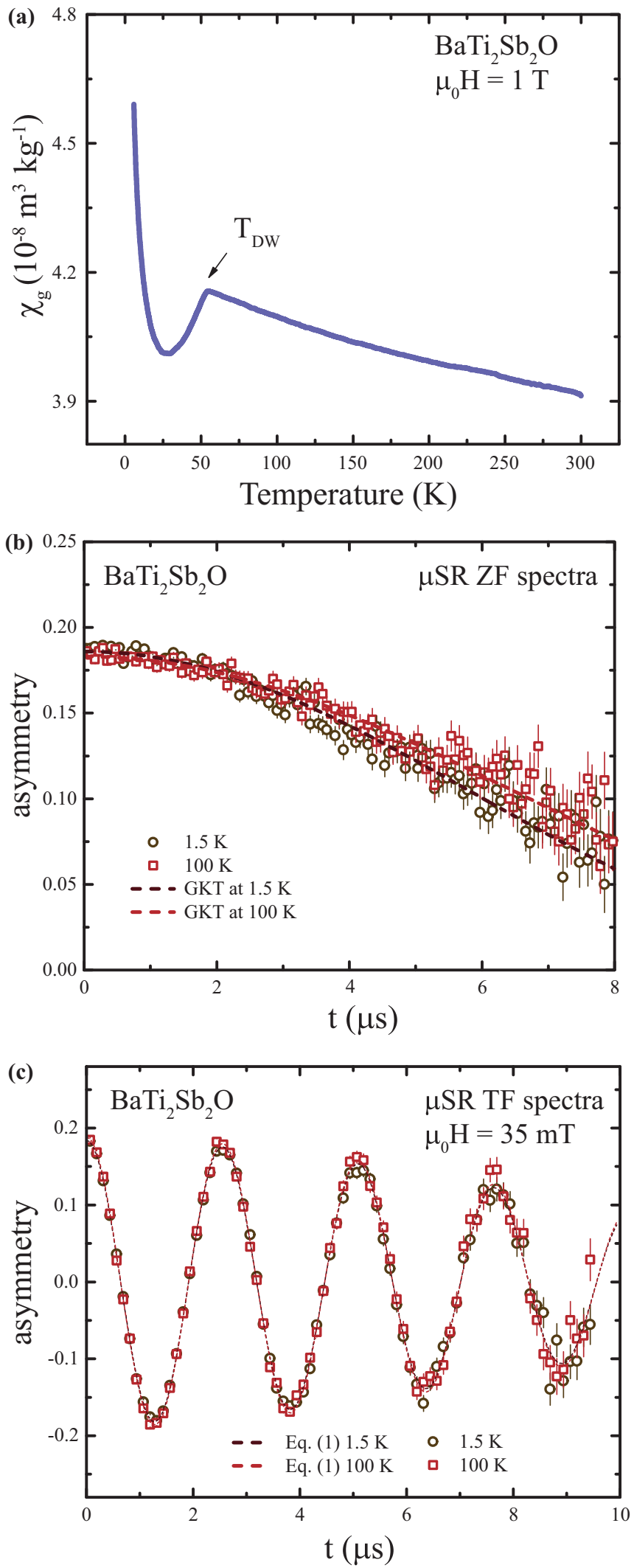

FIG. 1. (Color online) Parent compound, $\mathrm{BaTi}_{2} \mathrm{Sb}_{2} \mathrm{O}$, characterized by (A) the temperature-dependent susceptibility in a field of $\mu_{0} H=1 \mathrm{~T}$, (B) the ZF $\mu$ SR spectra at $1.5 \mathrm{~K}$ and $100 \mathrm{~K}$ (the dashed lines are fits to the Gaussian Kubo-Toyabe function), and (C) TF $\mu$ SR spectra (the dashed lines are fits to Eq. (1) at temperatures $T=1.5 \mathrm{~K}$ and $100 \mathrm{~K}$ ). down to $T=1.5 \mathrm{~K}$. Moreover, the relaxation rates are small and show only little differences between the measurements at high temperatures and at $1.5 \mathrm{~K}$. The $\mathrm{ZF}$ spectra are well described by a standard Gaussian Kubo-Toyabe (GKT) function, ${ }^{15}$ which is typical for nuclear moments. The zerofield $\mu$ SR spectra above and below $T_{\mathrm{DW}}$ do not exhibit any noticeable change in the relaxation rate, indicating the absence of a spontaneous internal field at the muon stopping site (within the sensitivity of $\mu \mathrm{SR}$ ). This is further supported by the weak TF measurements [Fig. 1(c)], where no reduction of the asymmetry is observed, as would be expected in case of magnetic ordering. Therefore, we can exclude that the observed transition at $T_{\mathrm{DW}}$ is caused by SDW ordering in the parent compound $\mathrm{BaTi}_{2} \mathrm{Sb}_{2} \mathrm{O}$. The observed transition is therefore most likely caused by CDW ordering. These findings are in agreement with recent NMR measurements. ${ }^{16}$

Upon substitution of barium by sodium in $\mathrm{Ba}_{1-x} \mathrm{Na}_{x} \mathrm{Ti}_{2} \mathrm{Sb}_{2} \mathrm{O}$, we find superconductivity with a maximum $T_{c}=5.1 \mathrm{~K}$ in the magnetization, and a bulk $T_{c \text {,bulk }}=4.5 \mathrm{~K}$ in the $\mu \mathrm{SR}$ measurements, for $x=0.25$. The temperature-dependent measurements of the DC magnetic susceptibility in the vicinity to superconductivity $(1.8 \mathrm{~K}$ to $10 \mathrm{~K})$, measured in zero-field-cooled (ZFC) mode in an external field of $\mu_{0} H=1 \mathrm{mT}$, are shown in Fig. 2(a). The transitions to the superconducting state are depicted for six representative members of the series, $x=0.05,0.1,0.15,0.2$, 0.25 , and 0.3. In Fig. 2(b) we show the ZF muon time signals for the optimally doped sample $x=0.25$ at $T=1.5 \mathrm{~K}$ and above $T_{c}(6 \mathrm{~K})$. The ZF spectra are well described by a GKT function and are overlapping for both measurements, revealing no magnetic ordering down to $1.5 \mathrm{~K}$. The relaxation above $T_{c}$ in the TF measurements in a field of $\mu_{0} H=35 \mathrm{mT}$ is shown in Fig. 2(c). Similar to the ZF measurements [Fig. 2(b)], only a small relaxation arising from the randomly aligned nuclear magnetic moments is observed. The strong additional relaxation in the TF measurements below $T_{c}$, however, is solely due to the formation of the flux-line lattice (FLL) in the Shubnikov phase. As shown by Brandt, the second moment of the resulting inhomogeneous field distribution is related to the magnetic penetration depth $\lambda$ as $\left\langle\Delta B^{2}\right\rangle \propto \sigma_{s c}^{2} \propto \lambda^{-4}$, whereas $\sigma_{s c}$ is the Gaussian relaxation rate due to the formation of the FLL. ${ }^{17,18}$ The TF $\mu$ SR time evolutions were analyzed using the following functional form for the polarization:

$$
A(t)=A(0) \exp \left[-\frac{\sigma_{s c}^{2}+\sigma_{n m}^{2}}{2} t^{2}\right] \cos \left(\gamma_{\mu} B_{\mathrm{int}} t+\varphi\right)+A_{\mathrm{BG}} \text {. }
$$

Here, $A(0)$ and $\varphi$ are the initial asymmetry and the phase of the muon ensemble, respectively, $\sigma_{n m}$ is the damping arising from the nuclear magnetic dipole moments, which we assumed to be temperature independent and fixed to the value obtained above $T_{c}, \gamma_{\mu} /(2 \pi)=135.5 \mathrm{MHz} / \mathrm{T}$ is the muon gyromagnetic ratio, and $B_{\text {int }}$ represents the internal magnetic field at the muon stopping site. For the low-temperature measurements in the LTF instrument, part of the muon beam is stopped in the silver sample holder, resulting in a background denoted as $A_{\mathrm{BG}}$.

For a weak-coupling BCS superconductor and $B_{\text {ext }} \ll B_{c 2}$, $\lambda$ does not depend on external magnetic fields, whereas, e.g., in 

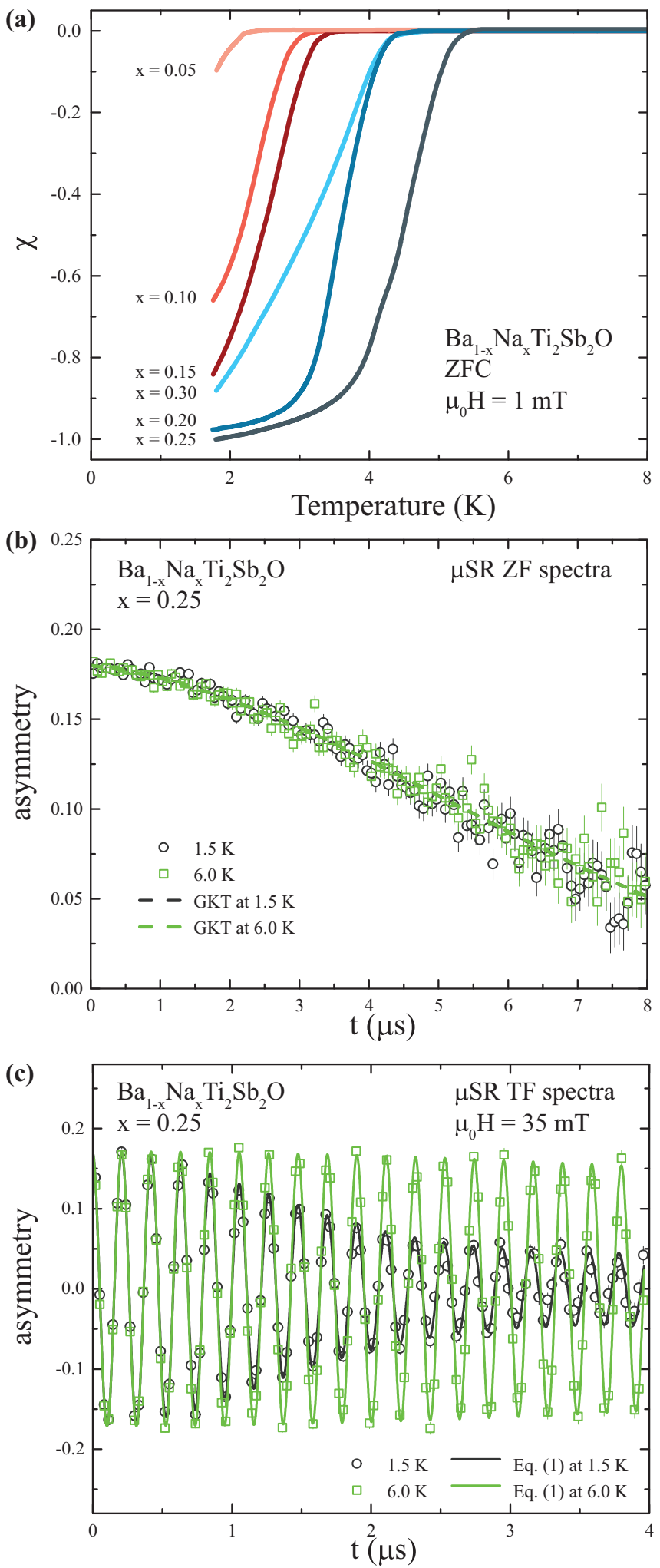

FIG. 2. (Color online) (A) The magnetic susceptibility of $\mathrm{Ba}_{1-x} \mathrm{Na}_{x} \mathrm{Ti}_{2} \mathrm{Sb}_{2} \mathrm{O}$ for $x=0.05,0.1,0.15,0.2,0.25,0.3$ measured in a field of $\mu_{0} H=1 \mathrm{mT}$. (B) The ZF $\mu \mathrm{SR}$ spectra for $x=0.25$ above $(6 \mathrm{~K})$ and below $(1.5 \mathrm{~K}) T_{c}$ (the dashed lines are the fits to the Gaussian Kubo-Toyabe function). (C) The TF $\mu$ SR spectra for $x=0.25$ above $(6 \mathrm{~K})$ and below $(1.5 \mathrm{~K}) T_{c}$ (the solid lines are fits to Eq. (1)). The strong relaxation of the signal at $1.5 \mathrm{~K}$ can be ascribed to the presence of the flux-line lattice.
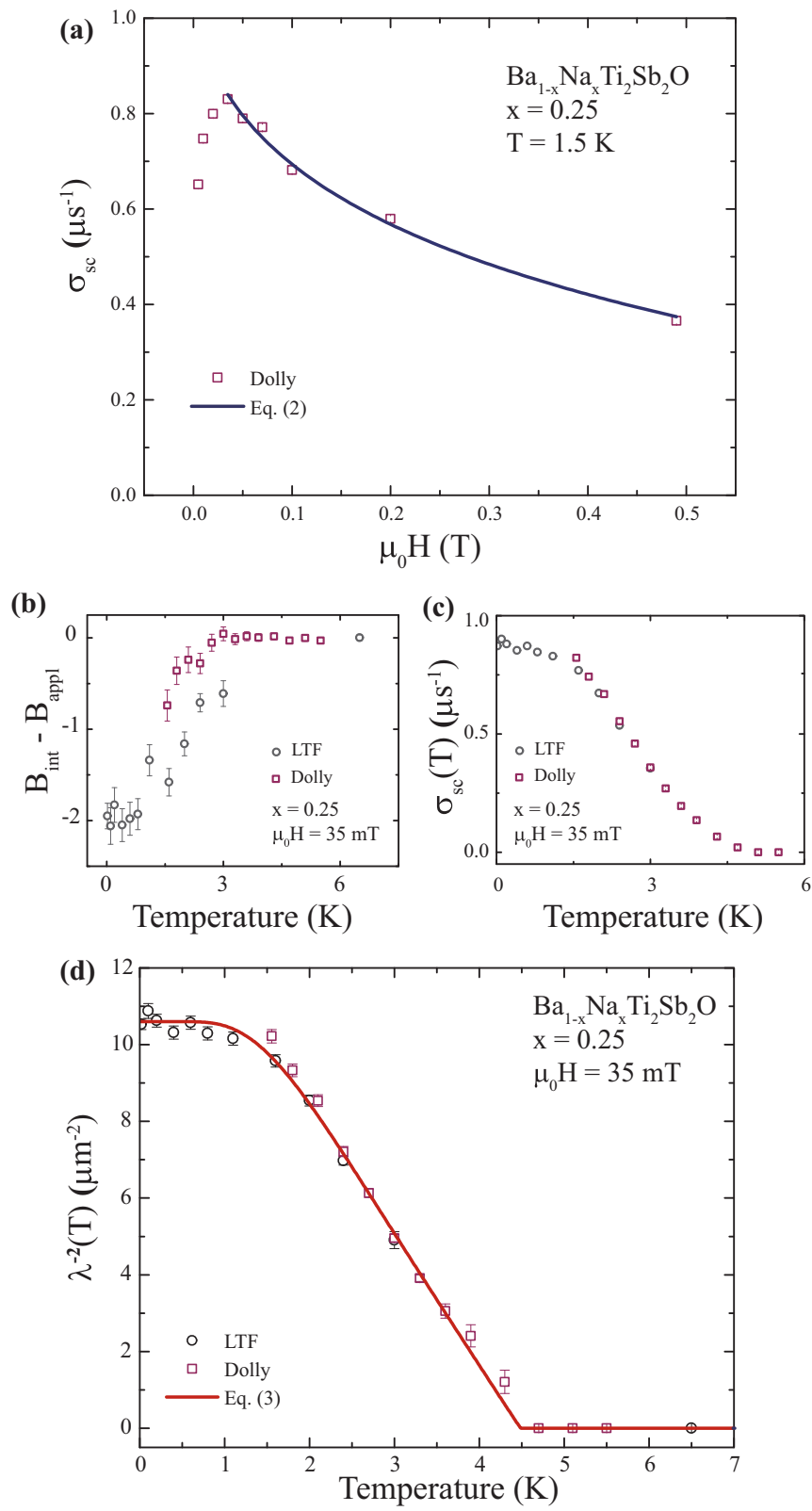

FIG. 3. (Color online) (A) Field dependence of the muon depolarization rate $\sigma_{s c}$ at $T \simeq 1.5 \mathrm{~K}$ for the optimal doping $x=0.25$. The solid line corresponds to a fit of the experimental data to Eq. (2). The insets show the corresponding field dependence of $\lambda^{-2}$ (in the Shubnikov phase). (B) The diamagnetic field shift in the superconducting state with respect to above $T_{c}\left(B_{\text {int }}-B_{\text {appl }}\right)$ for $x=$ 0.25 . (C) The temperature dependence of the muon polarization rate $\sigma_{s c}(T)$ measured in $\mu_{0} H=35 \mathrm{mT}$. (D) The temperature dependence of $\lambda^{-2}$ for $x=0.2$ as reconstructed from $\sigma_{s c}(T)$ (shown in $\mathrm{B}$ ), measured in $\mu_{0} H=35 \mathrm{mT}$. The solid line corresponds to a fit to Eq. (3) with $2 \Delta /\left(k_{B} T_{c}\right)=2.9$. Squares: Dolly instrument, circles: LTF instrument.

a multiple gap or nodal superconductor $\lambda$ can be significantly field dependent. ${ }^{19-22}$ In the case of an ideal vortex lattice of an isotropic $s$-wave superconductor within the Ginzburg-Landau theory, the relaxation rate $\sigma^{2}$ in the superconducting state 
should follow the expression ${ }^{18}$

$$
\sigma_{s c}=a\left(1-\frac{B}{B_{c 2}}\right)\left[1+1.21\left(1-\sqrt{\frac{B}{B_{c 2}}}\right)^{3}\right] \lambda^{-2} .
$$

Here, $a$ is a coefficient given by the symmetry of the vortex lattice (with $a=4.83 \times 10^{4} \mathrm{~nm}^{2} / \mu$ sec for triangular and $a=5.07 \times 10^{4} \mathrm{~nm}^{2} / \mu$ sec for a rectangular vortex lattice geometry $\left.{ }^{18,23}\right), B$ is the magnetic induction, for which we may assume $B \simeq B_{\text {ext }}$ in the region $\mu_{0} H_{c 1} \ll B_{\text {ext }} \ll \mu_{0} H_{c 2}$ (with $H_{c 1}$ the lower and $H_{c 2}$ the upper critical field, respectively). Equation (2) in general accounts for the reduction of $\sigma_{s c}$ due to the stronger overlap of the vortices with increasing field. A fit of the measured $\sigma_{s c}$ according to Eq. (2) describes the data reasonably well and yields $B_{c 2}=1.5(1) \mathrm{T}$ and $\lambda^{-2}=$ $10^{-5}(0.01) \mathrm{nm}^{-2}$ at $T=1.5 \mathrm{~K}$ for $x=0.25$ [see Fig. 3(a)]. The value of $B_{c 2}$, for the optimal doping $x=0.25$, is in excellent agreement with previous measurements. ${ }^{24}$ The parameter $a$ was fitted to $4.87(5) \times 10^{4} \mathrm{~nm}^{2} / \mu$ sec indicating that the vortex lattice in $\mathrm{Ba}_{1-x} \mathrm{Na}_{x} \mathrm{Ti}_{2} \mathrm{Sb}_{2} \mathrm{O}$ has triangular shape. To obtain maximum field contrast, we chose the magnetic field $\mu_{0} H=35 \mathrm{mT}$ to study the temperature dependence of $\lambda(T)$ for the optimally doped sample $x=0.25$. Measurements down to $T=0.02 \mathrm{~K}$ and $T=1.5 \mathrm{~K}$ were performed in the LTF and Dolly instruments, respectively. A diamagnetic shift of the internal magnetic field $B_{\text {int }}$ is observed below $T_{c}$ [Fig. 3(b)]. The resulting temperature dependence of $\sigma_{s c}$ is shown in Fig. 3(c). In Fig. 3(d) we show the temperature dependence of $\lambda^{-2}(T)$ as reconstructed from $\sigma_{s c}(T)$, using Eq. (2). The temperature dependence of $B_{c 2}(T)$, used in the corresponding calculation according to Eq. (2), was assumed to follow the theoretical Werthamer-Helfand-Hohenberg relation. ${ }^{24,25}$

These measurements suggest that $\lambda^{-2}$ is virtually temperature independent below $T \simeq 1 \mathrm{~K}$ for the optimally doped sample. The obtained experimental temperature dependence of $\lambda^{-2}(T)$ was tentatively analyzed within the clean limit approach for a London superconductor with an $s$-wave $\operatorname{gap}^{26}$

$$
\frac{\lambda^{-2}(T)}{\lambda^{-2}(0)}=1+2 \int_{\Delta(T)}^{\infty}\left(\frac{\partial f}{\partial E}\right) \frac{E}{\sqrt{E^{2}-\Delta^{2}(T)}} d E .
$$

Here $\lambda(0)$ is the zero-temperature value of the magnetic penetration depth, $f=\left[1+\exp \left(E / k_{B} T\right)\right]^{-1}$ is the Fermi function (with $k_{B}$ the Boltzmann constant), and $\Delta(T)=$ $\Delta(0) \tilde{\Delta}\left(T / T_{c}\right)$ represents the temperature dependence of the energy gap, which can be approximated to sufficient precision as $\tilde{\Delta}\left(T / T_{c}\right)=\tanh \left\{1.82\left[1.018\left(T_{c} / T-1\right)^{0.51}\right]\right\} .^{27}$ The results of this fit are $T_{c}=4.49(6) \mathrm{K}$ and $\Delta(0)=0.56(1) \mathrm{meV}$ with a zero-temperature magnetic penetration depth $\lambda=307(10) \mathrm{nm}$. This corresponds to a ratio $2 \Delta /\left(k_{B} T_{c}\right)=2.9$, which is quite close to the value of a weak-coupling BCS superconductor $2 \Delta /\left(k_{B} T_{c}\right)=3.5$. There are no signs of multigap superconductivity in these data (compare Refs. 19-22), and the presented low-temperature $\lambda^{-2}(T)$ data taken in a low magnetic field seem to be incompatible with a possible $d$-wave scenario. $^{28}$

\section{CONCLUSION}

We have presented magnetization and $\mu \mathrm{SR}$ results on the density wave (DW) ordering transition in $\mathrm{BaTi}_{2} \mathrm{Sb}_{2} \mathrm{O}$, and on the transition to superconductivity in $\mathrm{Ba}_{1-x} \mathrm{Na}_{x} \mathrm{Ti}_{2} \mathrm{Sb}_{2} \mathrm{O}$. The observed absence of a magnetic contribution to the $\mu \mathrm{SR}$ data related to the phase transition at $T_{\mathrm{DW}}=58 \mathrm{~K}$ of the parent compound $\mathrm{BaTi}_{2} \mathrm{Sb}_{2} \mathrm{O}$ is strong evidence against the SDW ordering transition theoretically proposed in Ref. 29. Therefore the observed phase transition is most likely due to CDW ordering that competes with a superconducting state. Upon substitution of barium by sodium in $\mathrm{Ba}_{1-x} \mathrm{Na}_{x} \mathrm{Ti}_{2} \mathrm{Sb}_{2} \mathrm{O}$ we find superconductivity with a maximum $T_{c}=5.1 \mathrm{~K}$ in the magnetization, and a bulk $T_{c \text {, bulk }}=4.5 \mathrm{~K}$ in the $\mu$ SR measurements, for $x=0.25$. In the TF $\mu$ SR spectra for $x=0.25$ below $T_{c}$ a strong relaxation of the signal is observed, which is due to the formation of the flux-line lattice. This is strong evidence for the bulk nature of the superconductivity in this material. The obtained experimental temperature dependence of $\lambda^{-2}(T)$ can be reasonably well explained within the clean limit approach for a conventional London superconductor, which is consistent with recently published NMR and specific-heat results, as well as theoretical calculations. ${ }^{16,24,30}$

\section{ACKNOWLEDGMENTS}

F.v.R. acknowledges a scholarship from Forschungskredit UZH, Grant No. 57161402. M.B. acknowledges the financial support of the Swiss National Science Foundation (Grant No. PBZHP2143495).

\footnotetext{
*vonrohr@physik.uzh.ch

${ }^{1}$ J. Paglione and R. L. Greene, Nat. Phys. 6, 645 (2010).

${ }^{2}$ H. Luetkens, H.-H. Klauss, M. Kraken, F. J. Litterst, T. Dellmann,

R. Klingeler, C. Hess, R. Khasanov, A. Amato, C. Baines,

M. Kosmala, O. J. Schumann, M. Braden, J. Hamann-Borrero, N. Leps, A. Kondrat, G. Behr, J. Werner, and B. Büchner, Nat. Mater. 8, 305 (2009).

${ }^{3}$ A. R. Moodenbaugh, Y. Xu, M. Suenaga, T. J. Folkerts, and R. N. Shelton, Phys. Rev. B 38, 4596 (1988).

${ }^{4}$ E. Morosan, H. W. Zandbergen, B. S. Dennis, J. W. G. Bos, Y. Onose, T. Klimczuk, A. P. Ramirez, N. P. Ong, and R. J. Cava, Nat. Phys. 2, 544 (2006).

${ }^{5}$ D. E. Moncton, J. D. Axe, and F. J. Di Salvo, Phys. Rev. Lett. 34, 734 (1975).
}

${ }^{6}$ L. F. Mattheiss and D. R. Hamann, Phys. Rev. Lett. 60, 2681 (1988).

${ }^{7}$ D. E. Cox and A. W. Sleight, Solid State Commun. 19, 969 (1976).

${ }^{8}$ J. Chang, E. Blackburn, A. T. Holmes, N. B. Christensen, J. Larsen, J. Mesot, Ruixing Liang, D. A. Bonn, W. N. Hardy, A. Watenphul, M. von Zimmermann, E. M. Forgan, and S. M. Hayden, Nat. Phys. 8, 871 (2012).

${ }^{9}$ D. Johrendt, H. Hosono, R.-D. Hoffmann, and R. Pöttgen, Z. Kristallogr. 226, 435 (2011)

${ }^{10}$ R. H. Liu, D. Tan, Y. A. Song, Q. J. Li, Y. J. Yan, J. J. Ying, Y. L. Xie, X. F. Wang, and X. H. Chen, Phys. Rev. B 80, 144516 (2009).

${ }^{11}$ A. Adam and H.-U. Schuster, Z. Anorg. Allg. Chem. 584, 150 (1990). 
${ }^{12}$ T. Yajima, K. Nakano, F. Takeiri, T. Ono, Y. Hosokoshi, Y. Matsushita, J. Hester, Y. Kobayashi, and H. Kageyama, J. Phys. Soc. Jpn. 82, 033705 (2013).

${ }^{13}$ P. Doan, M. Gooch, Z. Tang, B. Lorenz, A. Möller, P. C. W. Chu, and A. M. Guloy, J. Am. Chem. Soc. 134, 16520 (2012).

${ }^{14}$ A. Suter and B. Wojek, Physics Procedia 30, 69 (2012).

${ }^{15} \mathrm{R}$. Kubo and T. Toyabe, Magnetic Resonance and Relaxation (North-Holland, Amsterdam, 1967).

${ }^{16}$ S. Kitagawa, K. Ishida, K. Nakano, T. Yajima, and H. Kageyama, Phys. Rev. B 87, 060510 (2013).

${ }^{17}$ E. H. Brandt, Phys. Rev. B 37, 2349 (1988).

${ }^{18}$ E. H. Brandt, Phys. Rev. B 68, 054506 (2003).

${ }^{19}$ M. Bendele, S. Weyeneth, R. Puzniak, A. Maisuradze, E. Pomjakushina, K. Conder, V. Pomjakushin, H. Luetkens, S. Katrych, A. Wisniewski, R. Khasanov, and H. Keller, Phys. Rev. B 81, 224520 (2010).

${ }^{20}$ J. Sonier, J. Brewer, and R. Kiefl, Rev. Mod. Phys. 72, 769 (2000).
${ }^{21}$ R. Khasanov, A. Shengelaya, D. Di Castro, D. G. Eshchenko, I. M. Savić, K. Conder, E. Pomjakushina, J. Karpinski, S. Kazakov, and H. Keller, Phys. Rev. B 75, 060505 (2007).

${ }^{22}$ S. Serventi, G. Allodi, R. De Renzi, G. Guidi, L. Romano, P. Manfrinetti, A. Palenzona, Ch. Niedermayer, A. Amato, and Ch. Baines, Phys. Rev. Lett. 93, 217003 (2004).

${ }^{23}$ R. Khasanov, P. W. Klamut, A. Shengelaya, Z. Bukowski, I. M. Savic, C. Baines, and H. Keller, Phys. Rev. B 78, 014502 (2008).

${ }^{24}$ M. Gooch, P. Doan, Z. Tang, B. Lorenz, A. M. Guloy, and Paul C. W. Chu, arXiv:1308.1880.

${ }^{25}$ N. R. Werthamer, E. Helfand, and P. C. Hohenberg, Phys. Rev. 147, 295 (1966).

${ }^{26} \mathrm{M}$. Tinkham, Introduction to Superconductivity (Krieger Publishing Company, Malabar, FL, 1975).

${ }^{27}$ A. Carrington and F. Manzano, Physica C 385, 205 (2003).

${ }^{28}$ M. H. S. Amin, M. Franz, and Ian Affleck, Phys. Rev. Lett. 84, 5864 (2000).

${ }^{29}$ D. J. Singh, New J. Phys. 14, 123003 (2012).

${ }^{30}$ A. Subedi, Phys. Rev. B 87, 054506 (2013). 